\title{
EFEITO DA TERAPIA COM REALIDADE VIRTUAL NO EQUILÍBRIO DINÂMICO DE INDIVÍDUOS PÓS-ACIDENTE VASCULAR ENCEFÁLICO
}

Alice Haniuda Moliterno ${ }^{1}$, Isabela Bortolim Frasson ${ }^{1}$, Silas de Oliveira Damasceno ${ }^{1}$, Caroline Nunes Gonzaga ${ }^{1}$, Isabella Cristina Leoci ${ }^{1}$, Nicoly Ribeiro Uliam ${ }^{1}$, Isabella Menezes Silva ${ }^{1}$, Paola Larissa de Araujo Biazini ${ }^{1}$, João Pedro Lucas Neves Silva ${ }^{1}$, Rhuan Gustavo Duran Miron ${ }^{2}$, Guilherme Yassuyuki Tacao ${ }^{1}$, Lúcia Martins Barbatto ${ }^{1}$, Augusto Cesinando de Carvalho ${ }^{1}$.

${ }^{1}$ Universidade Estadual Paulista - UNESP, Presidente Prudente, SP; ${ }^{2}$ Universidade do Oeste Paulista - UNOESTE, Presidente Prudente, SP. e-mail: alice moliterno@hotmail.com

\section{RESUMO}

O objetivo desse estudo foi analisar o efeito da terapia com Realidade Virtual (RV) no equilíbrio dinâmico de indivíduos pós Acidente Vascular Encefálico (AVE). Participaram dez indivíduos com hemiparesia, que receberam 12 sessões individuais de fisioterapia com utilização da modalidade terapêutica RV, por meio do Nintendo Wii ${ }^{\circledR}$ Fit Plus e o Wii Sports Resort ${ }^{T M}$. A análise revelou que o instrumento Dynamic Gait Index (DGI), responsável por avaliar o equilíbrio dinâmico, obteve diferença significativa $(p=0,0085)$ entre a avaliação inicial $(17,30 \pm 3,59)$ e final $(20,30 \pm 2,94)$. $O$ jogo Penguin Slide não obteve significância entre as pontuações $(p=0,918)$, mas houve uma correlação moderada entre o instrumento $D G I(r=0,662 ; p=0,037)$. Esse estudo obteve resultados favoráveis relacionados ao equilíbrio dinâmico e também sugere que a terapia com RV como um todo influenciou a melhora do equilíbrio dinâmico dos indivíduos com hemiparesia pós-AVE.

Palavras-Chave: acidente vascular cerebral, equilíbrio postural, fisioterapia, realidade virtual, reabilitação.

\section{THE EFFECT OF VIRTUAL REALITY-BASED THERAPY ON DYNAMIC BALANCE TRAINING OF INDIVIDUALS AFTER STROKE}

\begin{abstract}
The aim of this study was to analyze the effect of virtual reality-based (VR) therapy on balance training of patients after stroke. 10 hemiparetic participants received twelve individual physical therapy sessions using the VR therapeutic, through Nintendo Wii ${ }^{\circledR}$ Fit Plus and Wii Sports Resort ${ }^{\mathrm{TM}}$. The analysis revealed that the Dynamic Gait Index (DGI) instrument, responsible for evaluating the dynamic balance, obtained significant difference $(p=0.0085)$ between the basal $(17.30 \pm 3.59)$ and final $(20.30 \pm 2.94)$ evaluation. The Penguin Slide game had no significance between the scores $(p=0.918)$, but there was a moderate correlation between the DGI instrument $(r=0.662 ; p=0.037)$. This study obtained favorable results related to dynamic balance and also suggests that VR therapy as in general influenced the improvement of dynamic balance in individuals with hemiparesis after stroke.
\end{abstract}

Keywords: stroke, postural balance, physical therapy specialty, virtual reality, rehabilitation.

\section{INTRODUÇÃO}

O Acidente Vascular Encefálico (AVE) apresenta grande relevância mundial por ser uma das principais causas de morte e incapacidade ${ }^{1-3}$.
Essa afecção neurológica geralmente compromete a funcionalidade dos indivíduos, pois podem ocorrer déficits motores, sensitivos, 
cognitivos e prejuízos nas habilidades de autocuidados e participação social ${ }^{2,4}$.

Dentre os comprometimentos do AVE pode-se enfatizar as alterações relacionadas ao equilíbrio, que afeta as atividades de vida diária, uma vez que, é capaz de limitar a marcha, as transferências, proporcionar risco de quedas, entre outros ${ }^{5,6}$.

Ainda, acredita-se na existência de um platô durante a recuperação após um AVE e comumente há um direcionamento da reabilitação para estágios mais agudos. Porém, cada vez mais evidências, revelam melhorias relacionadas a tarefas funcionais, mesmo em estágios crônicos ${ }^{2,7}$.

Diante disso, a fisioterapia expõe importante responsabilidade no tratamento dos indivíduos com sequelas de AVE, desde a minuciosa avaliação à escolha adequada dos recursos ou modalidades terapêuticas a serem utilizadas. $O$ tratamento fisioterapêutico muitas vezes se refere à busca pela manutenção ou restauração da funcionalidade e potencial máximo que pode ser atingido pelos indivíduos ${ }^{5,8}$. No entanto, geralmente 0 processo de recuperação desta população costuma ser longo, e pode se tornar desmotivador e difícil devido à repetitividade das atividades ${ }^{5}$.

Neste contexto, surgem novas modalidades terapêuticas com a finalidade de serem um meio alternativo ou complementar ${ }^{5,9}$, como a Realidade Virtual (RV). Essa terapia inovadora também denominada "Exergame" traz a tecnologia para o ambiente terapêutico, no qual, os participantes executam exercícios promovidos por jogos de vídeo game ${ }^{10,11}$. Há evidências que relatam favoráveis particularidades relacionados à $\mathrm{RV}$ para $\mathrm{a}$ reabilitação neurológica, como, a existência de repetições e objetivos durante as tarefas ${ }^{2,12,13}$, a possibilidade de intensificação da execução de prática simulada de $\operatorname{tarefas}^{2,14}$, a capacidade de proporcionar um ambiente enriquecido que viabiliza a resolução de problemas e aquisição de habilidades, com prática que frequentemente é mais agradável e desperta maior interesse ${ }^{2,15}$.

Porém, ainda é limitado e desconhecido a integralidade da eficácia clínica e dos possíveis efeitos de um programa de reabilitação com RV, o que torna pertinente a realização desse estudo, para maior conhecimento dos efeitos dessa modalidade terapêutica em indivíduos com sequelas de AVE. Portanto, o objetivo foi analisar o efeito da terapia com Realidade Virtual no equilíbrio dinâmico de indivíduos pós Acidente Vascular Encefálico.

\section{METODOLOGIA}

Esse estudo obteve a participação de indivíduos com sequelas de AVE atendidos em um centro de atendimentos em fisioterapia e reabilitação da UNESP. Todos os dez participantes foram previamente informados sobre os objetivos e procedimentos a serem adotados, e assinaram o termo de consentimento livre e esclarecido aprovado pelo Comitê de Ética da FCT-UNESP (CAAE: 90293618.5.0000.5402). Trata-se de um estudo clínico experimental, longitudinal, prospectivo e de braço único.

Foram incluídos indivíduos com hemiparesia pós-AVE, com encaminhamento médico, tempo de lesão igual ou superior a 12 meses, capazes de deambular com ou sem dispositivos auxiliares/órteses e com ausência de déficits cognitivos avaliados pelo Mini-exame do Estado Mental (ponto de corte para indivíduos analfabetos $18 / 19$ e para indivíduos com instrução escolar $24 / 25)^{16}$. Já os critérios para exclusão, consistiram em apresentação de, tempo de lesão inferior a 12 meses, dupla hemiparesia, afasia sensitiva ou condições de saúde adversas, tais como, outras doenças neurológicas ou ortopédicas não relacionadas ao AVE.

A princípio, realizou-se a coleta de dados pessoais e clínicos de cada participante e verificação dos critérios de inclusão. Posteriormente, foi realizada a avaliação inicial, que consistiu na aplicação do Dynamic Gait Index $(D G I)^{17,18}$, utilizado para avaliar o equilíbrio dinâmico dos indivíduos. Após seis semanas de intervenção, esse instrumento foi aplicado novamente, na avaliação final.

Os participantes desse estudo receberam doze sessões individuais de fisioterapia com utilização da modalidade terapêutica RV, sendo duas vezes por semana, durante seis semanas, e com duração de 45 min cada sessão. Antes de iniciarem a intervenção, receberam informações e instruções relacionadas aos equipamentos e jogos a serem utilizados, e em seguida foram submetidos a uma sessão inicial piloto, destinada apenas à familiarização do indivíduo com a RV.

No decorrer de cada sessão, os participantes foram dispostos em postura ortostática de frente para uma televisão. A pressão arterial e a frequência cardíaca foram verificadas no início e no final de cada sessão. 
Foram utilizados o Nintendo Wii ${ }^{\circledR}$ Fit Plus com o acessório Wii Balance Board, e o Wii Sports Resort $^{\mathrm{TM}}$ (Quadro 1).

O nível de dificuldade dos jogos foi determinado automaticamente de acordo com o desempenho de cada participante. O jogo Penguin Slide foi selecionado para análise neste estudo devido suas características específicas relacionadas ao equilíbrio.

Quadro 1. Descrição dos jogos utilizados.

\begin{tabular}{ll}
\hline Free Run & Caminhada livre com velocidade constante durante 5 min \\
\hline Hula Hoop & Solicita movimentos circulares com o quadril em uma base estável durante $70 \mathrm{~s}$ \\
\hline Perfect 10 & $\begin{array}{l}\text { Solicita movimentos com o quadril (sem mover os pés), simultaneamente a realização de } \\
\text { cálculos matemáticos, durante } 60 \mathrm{~s}\end{array}$ \\
\hline Penguin Slide & Solicita deslocamentos de peso para os lados, durante $90 \mathrm{~s}$ \\
\hline Free Step & $\begin{array}{l}\text { Consiste em subir e descer o step, que no caso, esta sendo representado pela plataforma } \\
\text { Wii Balance Board, durante } 5 \mathrm{~min}\end{array}$ \\
\hline Table Tennis & Jogo de tênis de mesa, durante $5 \mathrm{~min}$ \\
\hline \hline
\end{tabular}

Os dados foram analisados pelo software estatístico GraphPad Prism. Para análise de normalidade dos dados foi aplicado teste de Shapiro Wilk. Para análise pareada foi realizado teste $T$ de Student pareado para distribuição normal ou teste de Wilcoxon para distribuição não normal. Os dados foram expressos em média e desvio padrão. O coeficiente de correlação utilizado foi Correlação de Pearson $(r)$ para dados com distribuição normal. Para todas as análises foi considerado nível de significância de 5\%.

Também foi utilizado o Effect size (ES) pela fórmula Cohen $(d)$, que calcula o tamanho do efeito clínico. As leituras das magnitudes foram realizadas como efeito insignificante $(\geq 0,00$ a 0,15$)$; pequeno efeito $(\geq 0,15$ e $<0,40)$; médio efeito $(\geq 0,40$ e $<0,75)$; grande efeito $(>0,75)$. Esse conceito estatístico é traduzido normalmente pela diferença efetiva na população, sendo assim, quanto maior for o ES, maior será a manifestação do fenômeno na população ${ }^{19}$.

\section{RESULTADOS}

A amostra foi composta por dez indivíduos com hemiparesia, sendo cinco mulheres e cinco homens, com idade média de $64,4 \pm 9,54$ anos e tempo médio de lesão por AVE de $61,3 \pm 74,04$ meses. A pontuação média no Mini-exame do Estado Mental foi $26,8 \pm 3,35$ e a média da escolaridade foi de $8,2 \pm 4,10$ anos.

$O$ instrumento $\mathrm{DGI}$ apresentou uma média de $17,30 \pm 3,59$ pontos correspondentes à avaliação inicial e $20,30 \pm 2,94$ pontos correspondentes à avaliação final, realizada após - período de seis semanas destinado à intervenção. Ao realizar a comparação desses dois momentos analisados, revelou-se presença de diferença significativa (Figura 1). Além disso, a análise do Effect Size demonstrou grande efeito para o $D G I(d=0,96)$.

No entanto, ao analisar os resultados do jogo Penguin Slide (jogo inerente ao equilíbrio), obteve-se uma média inicial (primeira sessão) de $46,3 \pm 8,44$ pontos e final (décima segunda sessão) de $46,6 \pm 13,49$ pontos, e a comparação desses dois momentos não revelou melhora significativa (Figura 2).

Por fim, a análise da relação entre o instrumento DGI e o jogo Penguin Slide revelou uma correlação moderada $(r=0,662 ; p=0,037)$. 


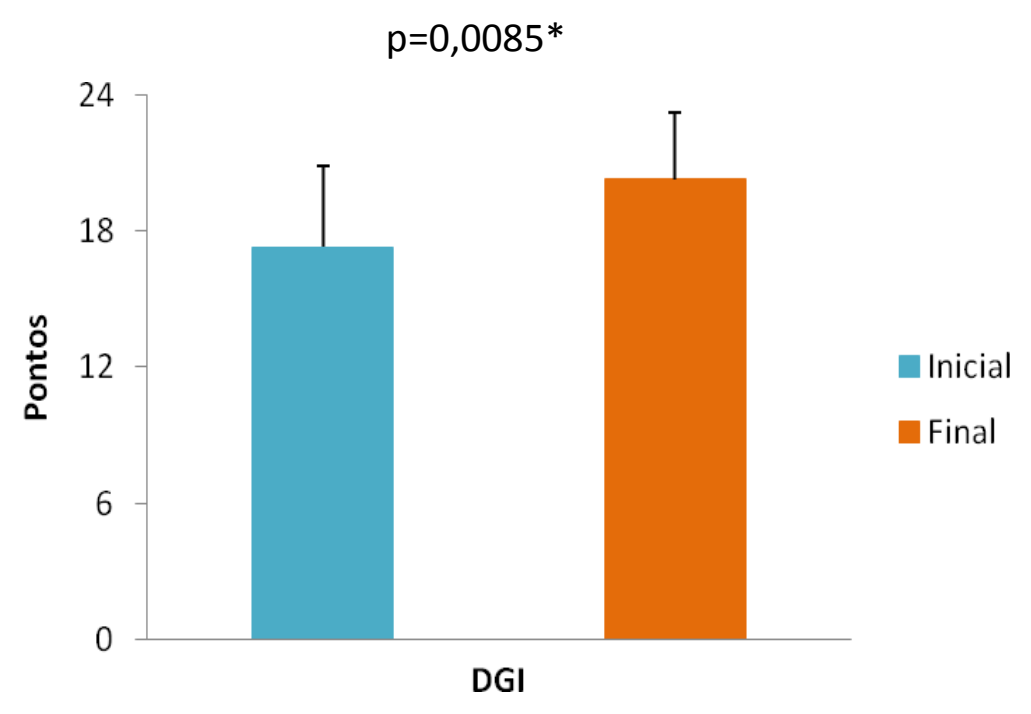

Figura 1. Representação gráfica do equilíbrio avaliado pelo instrumento DGI (média e desvio padrão). DGI= Dynamic Gait Index.

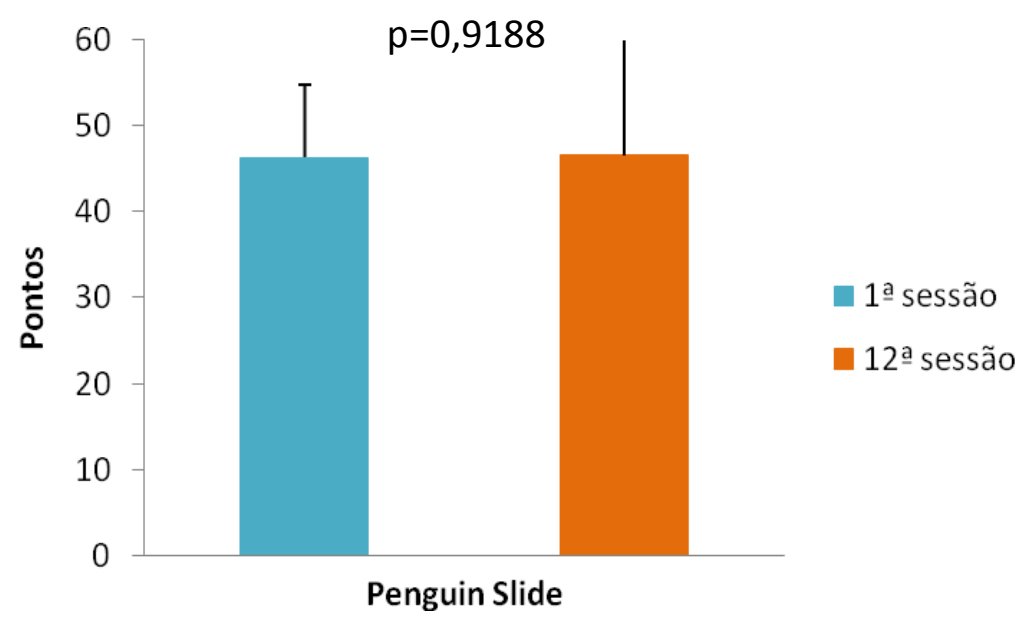

Figura 2. Representação gráfica da pontuação do jogo Penguin Slide (média e desvio padrão).

\section{DISCUSSÃO}

A tecnologia que se renova constantemente traz sensores atuais e inovadores, que denotam possibilidades de utilização dos Exergames como instrumento em programas de reabilitação, no qual se podem salientar, os treinos de equilíbrio ${ }^{20}$. Ainda, é possível a ocorrência do aumento da frequência de câmeras e softwares captadores de movimentos em programas de reabilitação, já que esses estão se tornando financeiramente mais acessíveis, com melhores precisões e qualidade de reconhecimento ${ }^{20,21}$. Diante disso, a relação da fisioterapia com a RV tem alcançado grandes proporções, e as investigações de seus possíveis impactos tem se tornado crescente.

Neste âmbito, podem-se destacar as observações a respeito do equilíbrio, já que esse apresenta participação crucial nas atividades rotineiras e de locomoção dos indivíduos, tornando seu processo de reabilitação essencial diante de patologias que acarretam o seu comprometimento $^{22,23}$. Deste modo, uma considerável quantidade de estudos constata benefícios no equilíbrio de indivíduos com hemiparesia, provenientes de reabilitações que contém feedback visual ${ }^{23,24}$ e isso pode ser explicado ao considerarmos que a informação visual fornecida, pode compensar os déficits somatossensoriais, resultando em uma melhor resposta motora ${ }^{23,25}$, argumentos esses que reforçam os achados adquiridos nesse estudo.

Ao analisar o efeito da terapia com RV sob o equilíbrio de indivíduos com sequelas crônicas de AVE, podemos constatar que esse tipo de treino após seis semanas, foi capaz de 
melhorar o equilíbrio dinâmico dos participantes, observado pelo instrumento DGl. Além disso, o Effect Size também demonstrou grande efeito clínico para o resultado desse instrumento. Assim, corroborando com outros pesquisadores que incentivam a utilização da RV para reabilitação do equilíbrio comprometido devido à sequela de $\operatorname{AVE}^{26}$.

Em um estudo de caso $^{27}$ foi observado um maior nível de restabelecimento funcional do equilíbrio, além de maior entusiasmo, no grupo que recebeu a intervenção por meio do Nintendo $\mathrm{Wii}^{\mathrm{TM}}$, em comparação ao tratamento convencional $^{27,28}$. Portanto, essas constatações citadas somam-se aos achados desse estudo, e enunciam a viabilidade de aplicação de protocolos com RV a benefício de tratamentos que envolvam o equilíbrio.

Também se estudou a relação entre o instrumento DGI e um dos jogos executados, classificado na categoria "Balance Games" e que recebe o nome "Penguin Slide", no qual foi obtida uma correlação moderada entre essas variáveis. Mas, vale ressaltar que, embora o DGI tenha evidenciado melhora significante em sua pontuação, o jogo analisado (Penguin Slide), não obteve diferença significativa entre a pontuação da primeira e última sessão. Desta forma, esses achados podem indicar que, o treino funcional em ambiente virtual como um todo foi capaz de melhorar o equilíbrio dinâmico de hemiparéticos crônicos e não apenas um jogo de forma isolada, mesmo que esse seja direcionado ao equilíbrio.

Desta forma, ao analisar o efeito da terapia com RV no equilíbrio de indivíduos pós AVE, concluiu-se que esta modalidade terapêutica possibilitou resultados favoráveis referente ao equilíbrio dinâmico, no qual se obteve significância e grande efeito clínico referente aos resultados do DGI. Também, revelou-se correlação moderada entre esse instrumento e o jogo Penguin Slide inerente ao equilíbrio, no qual, este último não adquiriu diferença significativa entre suas pontuações. Assim, sugere-se que a terapia com RV como um todo influenciou a melhora do equilíbrio dinâmico dos indivíduos participantes.

\section{CONFLITO DE INTERESSE}

Os autores declaram não haver qualquer potencial conflito de interesse que possa interferir na imparcialidade deste trabalho científico.

\section{REFERÊNCIAS}

1. Tally Z, Boetefuer L, Kauk C, Perez G, Schrand L, Hoder J. The efficacy of treadmill training on balance dysfunction in individuals with chronic stroke: a systematic review. Top Stroke Rehabil. 2017;24(7):539-46.

DOI: http://dx.doi.org/10.1080/10749357.2017.13454 $\underline{45}$

2. Laver KE, Lange B, George S, Deutsch JE, Saposnik G, Crotty M. Virtual reality for stroke rehabilitation. Cochrane Database of Syst Rev. 2017;11:CD008349.

DOI: http://dx.doi.org/10.1002/14651858.CD008349.p ub4

3. Feigin VL, Forouzanfar $M H$, Krishnamurthi $R$, Mensah GA, Connor M, Bennett DA et al. Global and regional burden of stroke during 1990-2010: findings from the Global Burden of Disease Study 2010. Lancet. 2014;383:245-55. DOI: http://dx.doi.org/10.1016/S0140-6736(13)61953$\underline{4}$

4. Miller EL, Murray L, Richards L, Zorowitz RD, Bakas T, Clark P et al. Comprehensive overview of nursing and interdisciplinary rehabilitation care of the stroke patient: a scientific statement from the American Heart Association. Stroke. 2010;41(10):2402-48. DOI: http://dx.doi.org/10.1161/STR.0b013e3181e7512 $\underline{b}$

5. Zheng QX, Ge L, Wang CC, Ma QS, Liao YT, Huang PP et al. Robot-assisted therapy for balance function rehabilitation after stroke: A systematic review and meta-analysis. Int J Nurs Stud. 2019;95:7-18. DOI: http://dx.doi.org/10.1016/j.ijnurstu.2019.03.015

6. Maeda N, Urabe Y, Murakami M, Itotani K, Kato J. Discriminant analysis for predictor of falls in stroke patients by using the Berg Balance Scale. Singapore Med J. 2015;56(5):280-3. DOI: http://dx.doi.org/10.11622/smedj.2015033

7. Teasell RW, Murie Fernandez M, McIntyre A, Mehta S. Rethinking the continuum of stroke rehabilitation. Arch Phys Med Rehabil. 2014;95(4):595-6. DOI: http://dx.doi.org/10.1016/j.apmr.2013.11.014

8. Hugues A, Di Marco J, Janiaud P, Xue Y, Pires J, Khademi $\mathrm{H}$ et al. Efficiency of physical therapy on 
postural imbalance after stroke: study protocol for a systematic review and meta-analysis. BMJ Open. 2017;7(1):e013348. DOI: http://dx.doi.org/10.1136/bmjopen-2016-013348

9. Lendraitienè $E$, Tamošauskaitè $A$, Petruševičienè $D$, Savickas R. Balance evaluation techniques and physical therapy in post-stroke patients: A literature review. Neurol Neurochir Pol. 2017;51(1):92-100. DOI: http://dx.doi.org/10.1016/j.pjnns.2016.11.003

10. Costa MTS, Vieira LP, Barbosa EO, Mendes Oliveira L, Maillot P, Ottero Vaghetti CA et al. Virtual Reality-Based Exercise with Exergames as Medicine in Different Contexts: A Short Review. Clin Pract Epidemiol Ment Health. 2019;15:15-20. DOI:

http://dx.doi.org/10.2174/174501790191501001 $\underline{5}$

11. Oh Y, Yang S. Meaningful play 2010 conference. Defining exergames \& exergaming, 2010.Proceedings. Disponível em: http:// meaningfulplay.msu.edu/ proceedings $2010 /$

12. Langhorne P, Bernhardt J, Kwakkel G. Stroke rehabilitation. Lancet 2011;377(9778):1693-702. DOI: $\quad$ https://doi.org/10.1016/S0140$\underline{6736(11) 60325-5}$

13. Veerbeek JM, Van Wegen E, Van Peppen R, Van der Wees PJ, Hendriks E, Rietberg $M$ et al. What is the evidence for physical therapy poststroke? A systematic review and metaanalysis. PLOS ONE. 2014;9(2):e87987. DOI: https://doi.org/10.1371/journal.pone.0087987

14. Demain S, Burridge J, Ellis-Hill C, Hughes AM, Yardley L, Tedesco-Triccas $L$ et al. Assistive technologies after stroke: self management or fending for yourself? A focus group study. BMC Health Serv Res. 2013;13:334. DOI: https://doi.org/10.1186/1472-6963-13-334

15. Lewis GN, Rosie JA. Virtual reality games for movement rehabilitation in neurological conditions: how do we meet the needs and expectations of the users? Disabil Rehabil. 2012;34(22):1880-6.

DOI: https://doi.org/10.3109/09638288.2012.670036
16. Bertolucci PHF, Brucki SMD, Campacci SR, Juliano Y. O Mini-Exame do Estado Mental em uma população geral: impacto da escolaridade. Arq Neuro-Psiquiatr. 1994;52(1):1-7. DOI: http://dx.doi.org/10.1590/S0004$\underline{\text { 282X1994000100001 }}$

17. Castro SM, Perracini MR, Ganança FF. Dynamic gait index - Brazilian version. Rev Bras Otorrinolaringol. 2006;72(6):817-25. DOI: http://dx.doi.org/10.1590/S00347299200600060 $\underline{0014}$

18. Jonsdottir J, Cattaneo D. Reliability and validity of the Dynamic Gait Index in persons with chronic stroke. Arch Phys Med Rehabil. 2007;88(11):1410-5. DOI: http://dx.doi.org/10.1016/j.apmr.2007.08.109

19. Loureiro LMJ, Gameiro MGH. Interpretação crítica dos resultados estatísticos: para lá da significância estatística. Rev Enferm Ref. 2011;3(3):151-62. DOI:

https://doi.org/10.12707/RIII1009

20. van Diest $M$, Lamoth C JC, Stegenga J, Verkerke G J, Postema K. Exergaming for balance training of elderly: state of the art and future developments. JNER. 2013;10:101. DOI: https://doi.org/10.1186/1743-0003-10-101

21. Lange B, Koenig S, Chang C-Y, McConnell E, Suma $E$, Bolas $M$ et al. Designing informed gamebased rehabilitation tasks leveraging advances in virtual reality. Disabil Rehabil. 2012;34:1863-70. DOI:

https://doi.org/10.3109/09638288.2012.670029

22. Nichols DS. Balance retraining after stroke using force platform biofeedback. Phys Ther. 1997;77(5):553-8.

DOI:

https://doi.org/10.1093/ptj/77.5.553

23. Ko $Y$, Ha H, Bae YH, Lee W. Effect of space balance 3D training using visual feedback on balance and mobility in acute stroke patients. J Phys Ther Sci. 2015;27(5):1593-6. DOI: https://doi.org/10.1589/ipts.27.1593

24. Van Peppen RP, Kortsmit M, Lindeman E, Kwakkel G. Effects of visual feedback therapy on postural control in bilateral standing after stroke: a systematic review. J Rehabil Med. 2006;38(1):3- 
9. DOI: https://doi.org/10.1080/16501970500344902

25. Mulder T, Hulstyn W. Sensory feedback therapy and theoretical knowledge of motor control and learning. Am J Phys Med. 1984;63(5):226-44.

26. Iruthayarajah J, McIntyre A, Cotoi A, Macaluso $S$, Teasell $R$. The use of virtual reality for balance among individuals with chronic stroke: a systematic review and meta-analysis. Top Stroke Rehabil. 2017;24(1):68-79. DOI: https://doi.org/10.1080/10749357.2016.1192361

27. Deutsch JE, Robbins D, Morrison J. Wii-based compared to standard of care balance and mobility rehabilitation for two individuals poststroke. Virtual Rehab Int Confer. 2009:117-20. DOI: https://doi.org/10.1109/icvr.2009.5174216

28. Mao Y, Chen P, Li L, Huang D. Virtual reality training improves balance function. Neural Regen Res. 2014;9(17):1628-34. DOI: https://doi.org/10.4103/1673-5374.141795 